\title{
Impacto de Erros Mecânicos e de Posicionamento do Detector na Dosimetria de Referência para Feixes em Modo FFF
} Impact Analysis for Detector Positioning and Mechanical Errors for Reference Dosimetry on FFF Beam Mode

\author{
Gustavo C. Panissi ${ }^{1}$, André V. Camargo ${ }^{1}$, Bruno Alvares ${ }^{1}$, Diego C. S. A. Silva ${ }^{1}$, Guilherme \\ A. Pavan, Gustavo D. Fioravante ${ }^{1}$, Lucas F. Guimarães, \\ Milena Giglioli, Thafarel Quaresma ${ }^{1}$
}

${ }^{1}$ Hospital de Amor - Fundação Pio XII - Barretos, Brasil

\begin{abstract}
Resumo
A implementação de novas tecnologias em radioterapia passou a aumentar o nível de exatidão exigido nos tratamentos. A calibração do feixe em dose absorvida na água é um importante indicador da acurácia na entrega da dose, já possuindo uma incerteza associada em suas medidas. O uso de feixes em modo FFF vem ganhando espaço em técnicas modernas, e devido a seus aspectos singulares, requerem uma melhor avaliação dos desvios envolvidos nesse processo de calibração. Diante disso, o trabalho procurou avaliar tais incertezas simulando erros de setup mecânicos e de posicionamento do detector, para diferentes configurações de feixe, taxa de dose e câmara de ionização. A análise foi feita de forma relativa, baseada no desvio percentual entre as leituras de carga fora e dentro das condições de referência. Os resultados foram apresentados sob três pontos de vista, fixando uma das variáveis de configuração e avaliando o maior desvio percentual obtido dentro de cada parâmetro. Ao final, foi feita uma avaliação geral do maior desvio obtido em cada parâmetro para todas as configurações de feixe, taxa de dose e detector. Os desvios em parâmetros mecânicos atingiram valores abaixo de 1,5\%, dentro dos limites toleráveis, porém altamente influenciados pelo volume do detector utilizado e pelo feixe. Erros de posicionamento do detector foram os mais afetados, com valores acima de $2,0 \%$ e atingindo seu maior valor para erros verticais $(3,6 \%)$. O perfil do feixe, a sensibilidade da câmara, a influência de fatores ligados à curva de PDP e influências no fator de recombinação iônica $\left(P_{\text {ion }}\right)$ ajudaram a explicar tais desvios. Com isso, ficou clara a importância de um controle de qualidade rígido em equipamentos que operam sob tal configuração, e os cuidados redobrados que devem ser tomados na montagem do setup experimental para a dosimetria de referência.
\end{abstract}

Palavras-chave: radioterapia; dosimetria; feixe FFF;

\begin{abstract}
The implementation of new technologies in radiotherapy has increased the accuracy level required in treatments. Absorbed dose calibration in water is an important indicator of the exactness on dose delivery, with an associated uncertainty on its measurements. FFF beam mode has been used in modern techniques, and due to singular aspects, require a better evaluation of deviations involved in the calibration process. Therefore, the work sought to evaluate such uncertainties by simulating mechanical setup and detector positioning errors, for different energy, dose rate and detector settings. The analysis was done in a relative way, based on the percentage deviation between the charge collection readings outside and within the reference conditions. The results were presented on three points of view, setting one of the configuration variables and evaluating the highest percentage deviation obtained within each parameter. At the end, a general analysis of the largest deviation obtained in each parameter was made for all energy, dose rate and detector configurations. The mechanical deviations reached values below $1.5 \%$, within tolerable limits but highly influenced by the volume of the detector and the energy. Positioning errors of the detector were the most affected, with values above $2.0 \%$ and reaching its highest for vertical errors (3.6\%). The beam profile, the chamber sensitivity, the influence of PDD curve settings and $P_{\text {ion }}$ influences has helped to explain such deviations. Thus, it became clear the importance of a rigid quality control in equipment operating under such mode, and the redoubled care that must be taken on experimental setup for reference dosimetry.
\end{abstract}

Keywords: radiotherapy, dosimetry, FFF Beam Mode.

\section{Introdução}

A radioterapia moderna se confronta cada dia mais com a necessidade critica em obter acurácia e precisão dentro do seu processo adaptativo clínico, passando desde as etapas de comissionamento e controle de qualidade, chegando às vias de tratamento do paciente. A evolução tecnológica trouxe consigo um novo conceito em exatidão, baseado em evidências clínicas através das curvas de dose-efeito, tanto para probabilidade de controle tumoral (TCP) quanto para probabilidade de complicação de tecidos normais (NTCP). Os grandes avanços recentes, entretanto, passaram a gerar preocupação sobre a estabilidade de tais 
curvas devido ao alto nível de acurácia que requerem. Estudos recentes ${ }^{1}$ mostraram que incertezas na dosimetria clínica, entre outros aspectos, podem provocar grandes mudanças nas regiões de intenso gradiente das curvas de doseefeito, podendo atingir de $10-20 \%$ para curvas de TCP e $20-30 \%$ para curvas de NTCP, sob um desvio de apenas $5 \%$ na entrega da dose.

Em meio a tal cenário, a utilização de feixes de radiação de alta energia foi sempre objeto de um rígido programa de controle de qualidade do aparelho, em conformidade com limites já préestabelecidos através de protocolos internacionais ${ }^{2}$. Os processos para a garantia da qualidade incluem a calibração do feixe em termos da dose absorvida na água, um importante indicador da acurácia do equipamento na entrega da dose ao paciente. $O$ protocolo TRS-398 da Agência Internacional de Energia Atômica (IAEA) ${ }^{3}$ é o documento padrão a ser seguido durante a calibração, e estabelece condições ideais de medidas para diversos tipos de feixes. Em se tratando de fótons de alta energia, o protocolo especifica as condições de referência utilizando setup SSD e tamanho de campo 10x10 $\mathrm{cm}^{2}$, usando câmara de ionização preferencialmente cilíndrica a $10 \mathrm{~cm}$ de profundidade em água e disposta com seu volume sensível alinhado com o eixo central do feixe. O TRS-398 estima que em todo o processo de calibração, dois tipos de incertezas acabam surgindo inevitavelmente, sendo a primeira delas associada à calibração do detector em termos do $N_{D, w}$, e a segunda nas etapas de medidas no ponto de referência dentro do fantoma de água. De uma incerteza combinada total de $1,5 \%$, apenas o estabelecimento das condições de referência já citadas contribui com um desvio padrão relativo de $0,4 \%$.

As recomendações citadas pelo TRS-398 tomam como base equipamentos que fazem uso do filtro aplanador, cujo sistema é capaz de gerar uma distribuição de dose uniforme ao longo de toda a extensão do campo de tratamento. Entretanto, os grandes debates em radioterapia passaram a visar o desenvolvimento de técnicas mais robustas que passassem a unir redução no tempo de tratamento e maior conformação ao volume alvo, com evidente redução de toxicidade em tecidos sadios. O uso de técnicas modificadoras de fluência e sob condições estereotáxicas, tais como a Radioterapia de Intensidade Modulada (IMRT) e a Radiocirurgia (SRS), passaram a eliminar a necessidade do uso do filtro aplanador, levando ao surgimento de uma nova geração de equipamentos operando sob o modo FFF (Flattening Filter Free). Os feixes FFF, além de operarem sob uma maior taxa de dose, eliminam o aspecto planar do feixe ao longo do campo, gerando um perfil de dose típico com elevado gradiente de dose fora do eixo central.

Assim como ocorre para feixes convencionais, os aparelhos com sistema FFF também são alvo de um rígido programa de controle de qualidade, e sob o aspecto de calibração da dose na água exigem uma atenção especial quanto aos processos envolvidos.
Em primeiro lugar, a alta dose por pulso leva a um aumento do fator de recombinação iônica do feixe $\left(P_{\text {ion }}\right)$, gerando uma redução na eficiência de coleta de carga pela câmara de ionização. Além de provocar mudanças significativas nas medidas de porcentagem em dose profunda (PDP) e do fator Off-Axis (OAR) ${ }^{4}$, o adendo ao TG- $51^{5}$ ressalta ainda mudanças no formalismo do fator de qualidade $\mathrm{K}_{\mathrm{Q}, \mathrm{Q}}$ para feixes FFF. Além disso, o tamanho do detector utilizado pode vir a provocar efeitos de Volume Averaging, levando a uma subestimação da dose no eixo central de até $0,2 \%$ em sua leitura, sendo que alguns estudos ${ }^{6}$ propuseram o uso de um fator de correção $\mathrm{P}_{\mathrm{rp}}$ para corrigir tal efeito. O Report da $A A P M$ para feixes $\mathrm{FFF}^{7}$ recomenda um maior cuidado durante o posicionamento da câmara no ponto de referência, e revela que um desvio de até 5 $\mathrm{mm}$ fora do eixo central poderia levar a uma diminuição de até $0,3 \%$ da leitura real a ser mensurada.

Através da definição de acurácia, e levando em conta as singularidades contidas no feixe FFF e as definições de incertezas estabelecidas pelo TRS398, houve uma preocupação em se estudar a fundo os aspectos dosimétricos desse sistema. Diante disso, o objetivo desse trabalho consiste em avaliar o impacto na dosimetria de referência de feixes FFF quando são introduzidas variações mecânicas e de posicionamento do detector fora daquelas recomendadas pelo TRS-398.

\section{Materiais e Métodos}

Para o estudo em questão, foi utilizado um objeto simulador de água de dimensões $40 \times 40 \times 40 \mathrm{~cm}^{3} \mathrm{e}$ eletrômetro Standard Imaging modelo CDX 2000B. As medidas foram realizadas no acelerador linear TrueBeam ${ }^{\circledR}$ STX (Varian Medical Systems, Palo Alto, CA), através dos feixes de 6MV FFF e $10 \mathrm{MV}$ FFF. As condições de referência segundo o TRS398 foram rigorosamente determinadas por testes mecânicos no aparelho. A montagem do setup foi feita segundo as recomendações do protocolo, com gantry e colimador a $0^{\circ}$, tamanho de campo $10 \times 10$ $\mathrm{cm}^{2}$ e SSD $=100 \mathrm{~cm}$, com a câmara posicionada no centro do reticulado e a $10 \mathrm{~cm}$ de profundidade. Duas câmaras de ionização foram usadas para o estudo, de forma a se avaliar o impacto do volume do detector nas medidas e os possíveis efeitos de sensibilidade a erros: câmara de ionização tipo Farmer FC65-G de 0,65 cm³ (Iba, Scanditronix, Wellhofer) e câmara de ionização Compact CC13 de $0,13 \mathrm{~cm}^{3}$ (Iba, Scanditronix, Wellhofer). Para cada câmara e feixe, a taxa de dose foi variada tomandose uma delas como padrão (400 UM/min) e a outra como a maior disponível para o feixe utilizado (1400 $\mathrm{UM} / \mathrm{min}$ para 6MV FFF e $2400 \mathrm{UM} /$ min para 10MV FFF), de forma a se avaliar possíveis impactos na coleta de carga pela $\mathrm{Cl}$. Após a montagem do setup, e dentro de cada configuração apresentada, três medidas a $-300 \mathrm{~V}$ e 100 UM cada foram realizadas a fim de se obter uma melhor amostragem dos dados. A disposição dos feixes e das taxas de doses 
utilizadas para cada detector estão resumidas na Tabela 1 abaixo:

Tabela 1: Configurações de feixe e taxa de dose no aparelho, utilizadas para cada detector;

\begin{tabular}{ccc}
\multicolumn{3}{c}{ utilizadas para cada detector; } \\
\hline Detector & Feixe (MV) & Taxa de Dose (UM/min) \\
\hline 6 FFF & $400 / 1400$
\end{tabular}

FC65-G / CC13

10 FFF

$400 / 2400$

Assim, após a obtenção dos dados sob as condições de referência, foram simuladas variações mecânicas no aparelho e erros de posicionamento do detector, coletando-se os dados sob cada erro de setup introduzido. A tabela 2 ilustra os parâmetros variados e os deslocamentos fora das condições de referência introduzidos sobre eles:

Tabela 2. Condições de Referência (TRS-398) e variações dos parâmetros, simulando possíveis erros na montagem do setup;

\begin{tabular}{ccc}
\hline Parâmetros & Referência & Erros de Setup \\
\hline Gantry $\left(^{\circ}\right)$ & 0 & $-1,0 ;-0,5 ;+0,5 ;+1,0$ \\
Colimador $\left(^{\circ}\right)$ & 0 & $-1,0 ;-0,5 ;+0,5 ;+1,0$ \\
Tamanho de Campo & 10 & 9,$6 ; 9,8 ; 10,2 ; 10,4$ \\
$\left(\mathrm{~cm}^{2}\right)$ & & \\
SSD $(\mathrm{cm})$ & 100 & 99,$8 ; 99,9 ; 100,1 ; 100,2 ;$ \\
$\begin{array}{c}\text { Posição Lateral da Cl } \\
(\mathrm{X}-\mathrm{cm})\end{array}$ & 0 & $-1,0 ;-0,5 ;-0,25 ;+0,25 ;$ \\
$\begin{array}{c}\text { Posição Vertical da } \\
\text { Cl }(\mathrm{Y}-\mathrm{cm})\end{array}$ & 0 & $-0,5 ;-0,25 ;+0,25 ;+0,5$ \\
$\begin{array}{c}\text { Posição Longitudinal } \\
\text { da Cl }(Z-\mathrm{cm})\end{array}$ & 0 & $-1,0 ;-0,5 ;-0,25 ;+0,25 ;$ \\
\hline
\end{tabular}

Assim, a análise dos dados foi feita de forma relativa, tomando-se as leituras de carga (nC) simuladas com erros de setup e as normalizando segundo as leituras obtidas nas condições de referência. Com isso, foi possível calcular o erro percentual relativo (\%) das medidas e assim mensurar se tais desvios dosimétricos se mantém em conformidade com os padrões de tolerância já estabelecidos para a dosimetria de referência segundo o TG142 e o TRS-398 (2\% Mensal e 3\% Diário), segundo a equação abaixo:

$$
\operatorname{Desvio}(\%)=\frac{100 \times\left(L_{i}-L_{R}\right)}{L_{R}}
$$

Onde $L_{i}$ e $L_{R}$ representam, respectivamente, as médias das leituras em carga $(\mathrm{nC})$ obtidas fora e dentro das condições de referência. Temperatura e pressão foram monitoradas constantemente durante a coleta de dados, a fim de se avaliar a manutenção das mesmas condições para todas as medidas e evitar possíveis influências no fator $\mathrm{k}_{\mathrm{T}, \mathrm{P}}$.

\section{Resultados}

Os resultados descritos abaixo tomam como base o maior desvio percentual (\%) encontrado dentro de cada um dos parâmetros avaliados (Gantry, Colimador, Campo, SSD, Posição do Detector Lateral, Vertical e Longitudinal), seja representando um aumento ou seja mostrando uma diminuição dos dados coletados nas condições ideais.

As análises foram divididas em quatro etapas: a primeira delas toma como base os detectores, e dentro de cada taxa de dose utilizada avalia em qual feixe ocorreu o maior desvio percentual. As Figuras 1 e 2 e a Tabela 3 representam essa situação:

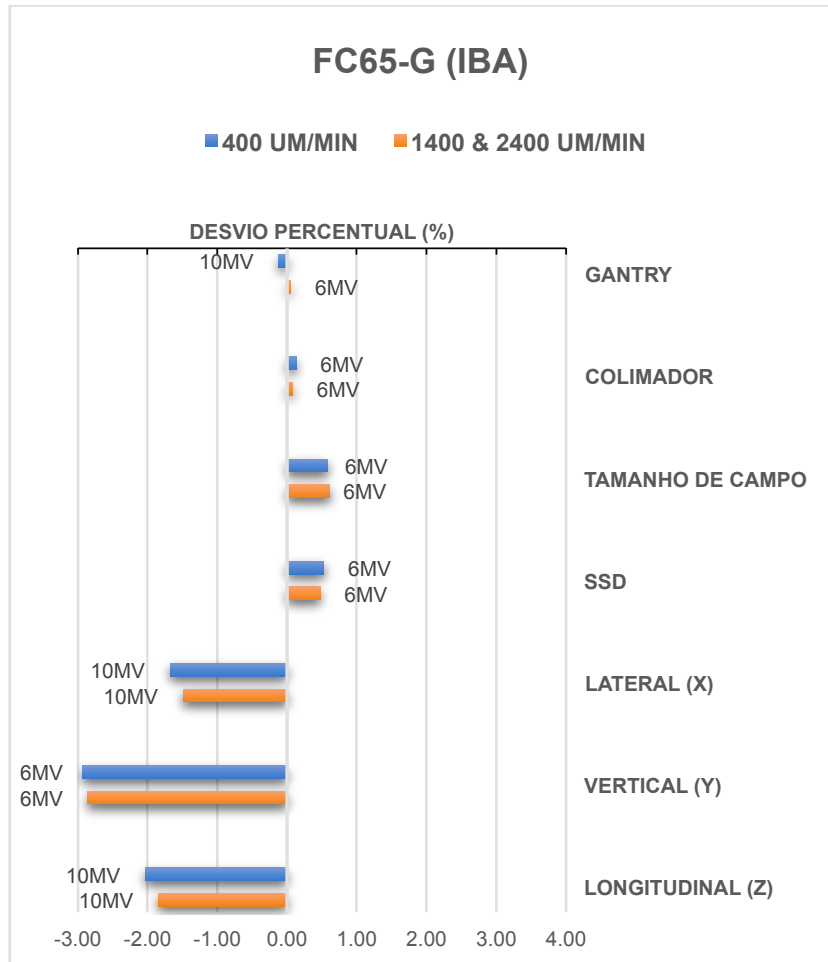

Figura 1. Desvio percentual máximo (\%) encontrado em cada parâmetro, comparando-se duas taxas de dose para a câmara FC65;

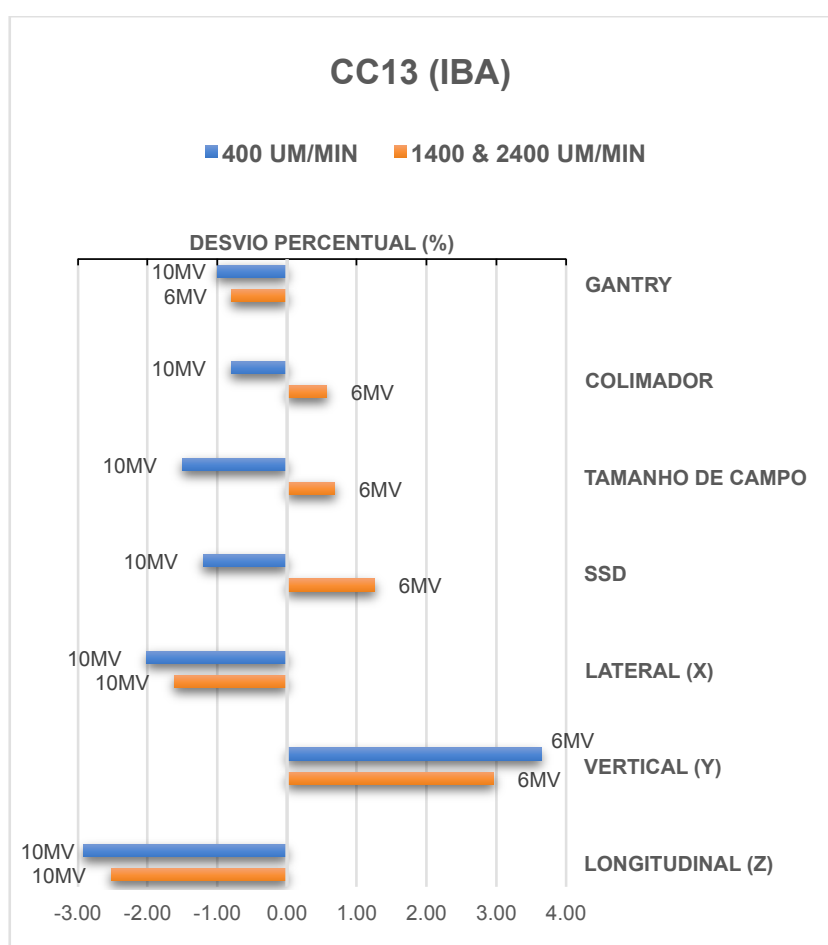

Figura 2. Desvio percentual máximo (\%) encontrado em cada parâmetro, comparando-se duas taxas de dose para a câmara CC13; 
Tabela 3. Desvios percentuais máximos, para cada parâmetro avaliado dentro dos detectores:

\begin{tabular}{ccccc}
\hline Detector & \multicolumn{2}{c}{ FC65-G (IBA) } & \multicolumn{2}{c}{ CC13 (IBA) } \\
\hline $\begin{array}{c}\text { Taxa de Dose } \\
(\text { UM/min) }\end{array}$ & 400 & 1400 & 400 & 1400 \\
& \multicolumn{3}{c}{ Desvio Percentual (\%) } \\
\hline & \multicolumn{3}{c}{0400} \\
\hline Gantry $\left(^{\circ}\right)$ & $-0,118$ & 0,053 & $-1,002$ & $-0,797$ \\
Colimador $\left(^{\circ}\right)$ & 0,132 & 0,079 & $-0,802$ & 0,569 \\
Campo $\left(\mathrm{cm}^{2}\right)$ & 0,582 & 0,609 & $-1,503$ & 0,683 \\
SSD $(\mathrm{cm})$ & 0,529 & 0,477 & $-1,202$ & 1,253 \\
Lateral $(\mathrm{X})(\mathrm{cm})$ & $-1,675$ & $-1,489$ & $-2,010$ & $-1,615$ \\
Vertical $(\mathrm{Y})(\mathrm{cm})$ & $-2,939$ & $-2,864$ & 3,641 & 2,961 \\
Longitudinal $(\mathrm{Z})(\mathrm{cm})$ & $-2,029$ & $-1,844$ & $-2,915$ & $-2,523$ \\
\hline
\end{tabular}

Para o detector FC65-G, as posições onde ocorreram os maiores desvios para 400 e 1400/2400 $\mathrm{UM} / \mathrm{min}$ foram, respectivamente: $-0,5^{\circ} \mathrm{e}-1,0^{\circ}$ para Gantry; $+0,5^{\circ}$ e $-1,0^{\circ}$ para Colimador; $10,4 \mathrm{~cm}^{2}$ e $10,4 \mathrm{~cm}^{2}$ para Tamanho de Campo; $99,8 \mathrm{~cm}$ e 99,8 $\mathrm{cm}$ para SSD; $-1,0 \mathrm{~cm}$ e $-1,0 \mathrm{~cm}$ para ambas as posições $\mathrm{X} \mathrm{e} \mathrm{Z;}+0,5 \mathrm{~cm}$ e $+0,5 \mathrm{~cm}$ para posição $\mathrm{Y}$;

Para o detector CC13, as posições onde ocorreram os maiores desvios para 400 e 1400/2400 UM/min foram, respectivamente: $-1,0^{\circ} \mathrm{e}-1,0^{\circ}$ para Gantry; $+0,5^{\circ}$ e $-0,5^{\circ}$ para Colimador; $9,6 \mathrm{~cm}^{2}$ e 10,4 $\mathrm{cm}^{2}$ para Tamanho de Campo; $100,2 \mathrm{~cm}$ e $99,8 \mathrm{~cm}$ para SSD; $-1,0 \mathrm{~cm}$ e $-1,0 \mathrm{~cm}$ para ambas as posições $X$ e Z; $-0,5 \mathrm{~cm}$ e $-0,5 \mathrm{~cm}$ para posição $Y$;

A segunda análise toma como base a mesma taxa de dose, e dentro de cada feixe utilizado avalia em qual detector ocorreu o maior desvio percentual. As Figuras 3 e 4 e a Tabela 4 representam essa situação:

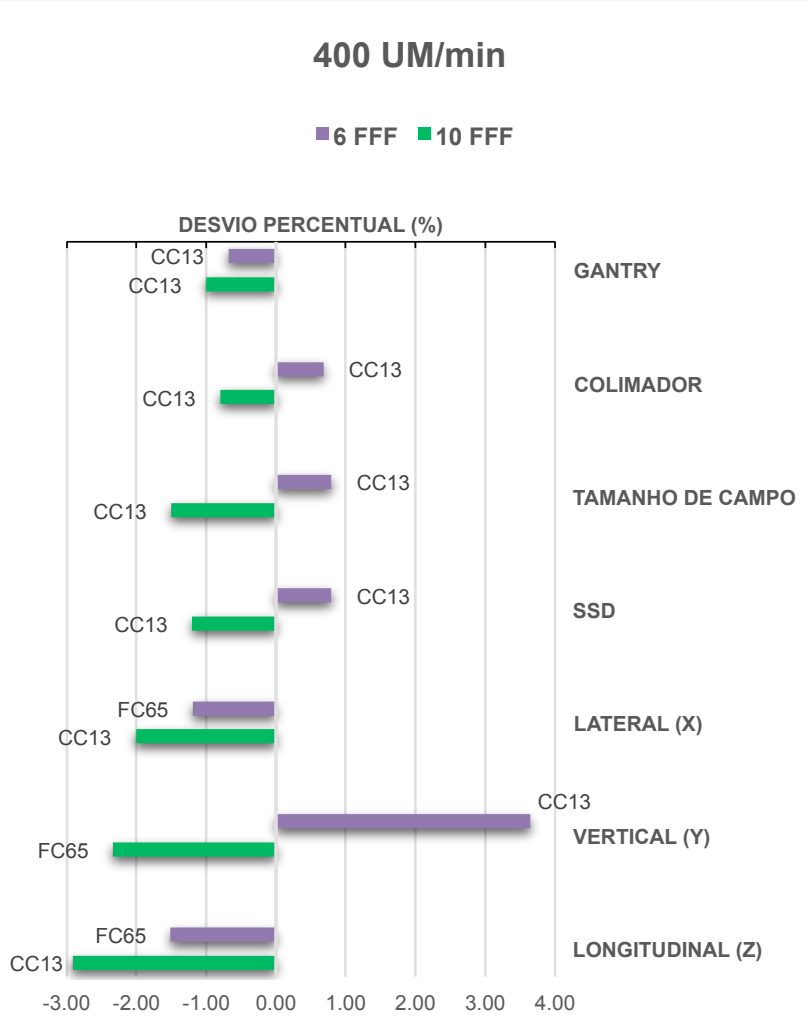

Figura 3. Desvio percentual máximo (\%) encontrado em cada parâmetro, comparando-se dois tipos de feixe para a taxa de dose de $400 \mathrm{UM} / \mathrm{min}$;

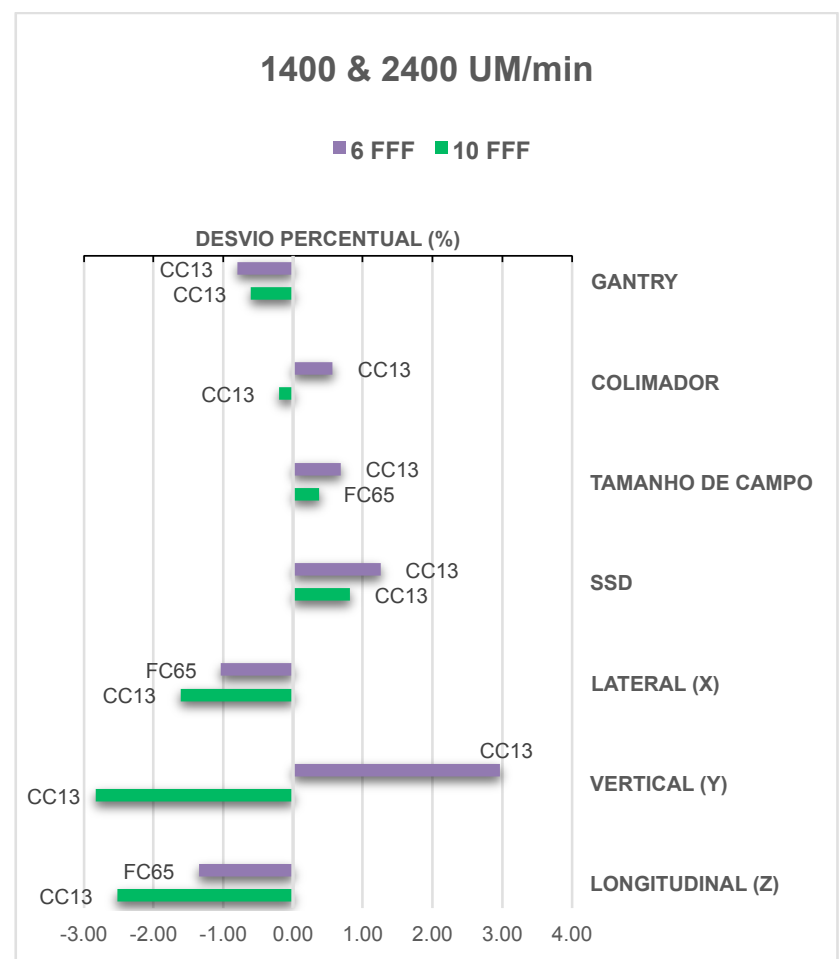

Figura 4. Desvio percentual máximo (\%) encontrado em cada parâmetro, comparando-se dois tipos de feixe para as taxas de dose de 1400 / 2400 UM/min;

Tabela 4. Desvios percentuais máximos, para cada parâmetro avaliado dentro das taxas de dose

\begin{tabular}{cclrr}
\hline $\begin{array}{c}\text { Taxa de Dose } \\
(\mathrm{UM} / \mathrm{MIN})\end{array}$ & \multicolumn{3}{c}{400} & \multicolumn{3}{c}{1400 \& 2400 } \\
\hline Feixe (MV) & 6 FFF & 10 FFF & 6 FFF & $10 \mathrm{FFF}$ \\
\hline & \multicolumn{4}{c}{ Desvio Percentual (\%) } \\
\hline Gantry $\left(^{\circ}\right)$ & $-0,683$ & $-1,002$ & $-0,797$ & $-0,607$ \\
Colimador $\left(^{\circ}\right)$ & 0,683 & $-0,802$ & 0,569 & $-0,202$ \\
Campo $\left(\mathrm{cm}^{2}\right)$ & 0,796 & $-1,503$ & 0,683 & 0,378 \\
SSD $(\mathrm{cm})$ & 0,796 & $-1,202$ & 1,253 & 0,810 \\
Lateral $(\mathrm{X})(\mathrm{cm})$ & $-1,191$ & $-2,010$ & $-1,034$ & $-1,615$ \\
Vertical $(\mathrm{Y})(\mathrm{cm})$ & 3,641 & $-2,336$ & 2,961 & $-2,825$ \\
Longitudinal $(\mathrm{Z})(\mathrm{cm})$ & $-1,509$ & $-2,915$ & $-1,352$ & $-2,523$ \\
\hline
\end{tabular}

Para a taxa de dose de $400 \mathrm{UM} / \mathrm{min}$, as posições onde ocorreram os maiores desvios para $6 \mathrm{MV}$ FFF e 10 MV FFF foram, respectivamente: $-1,0^{\circ} \mathrm{e}-1,0^{\circ}$ para Gantry; $1,0^{\circ} \mathrm{e}+0,5^{\circ}$ para Colimador; $10,4 \mathrm{~cm}^{2} \mathrm{e}$ 9,6 $\mathrm{cm}^{2}$ para Tamanho de Campo; 99,8 cm e 100,2 $\mathrm{cm}$ para SSD; $-1,0 \mathrm{~cm}$ e $-1,0 \mathrm{~cm}$ para ambas as posições $\mathrm{X}$ e Z; $-0,5 \mathrm{~cm}$ e $+0,5 \mathrm{~cm}$ para posição $\mathrm{Y}$;

Para a taxa de dose de $1400 / 2400 \mathrm{UM} / \mathrm{min}$, as posições onde ocorreram os maiores desvios para 6 MV FFF e 10 MV FFF foram, respectivamente: $1,0^{\circ} \mathrm{e}-0,5^{\circ}$ para Gantry; $-0,5^{\circ}$ e $+0,5^{\circ}$ para Colimador; $10,4 \mathrm{~cm}^{2}$ e $10,4 \mathrm{~cm}^{2}$ para Tamanho de Campo; 99,8 cm e 99,8 cm para SSD; $-1,0 \mathrm{~cm} \mathrm{e} \mathrm{-1,0}$ $\mathrm{cm}$ para ambas as posições $X$ e $Z ;-0,5 \mathrm{~cm} \mathrm{e}+0,5$ $\mathrm{cm}$ para posição $\mathrm{Y}$;

A terceira análise toma como base o mesmo feixe, e dentro de cada detector utilizado avalia em qual taxa de dose ocorreu o maior desvio percentual. As Figuras 5 e 6 e a Tabela 5 representam essa situação: 


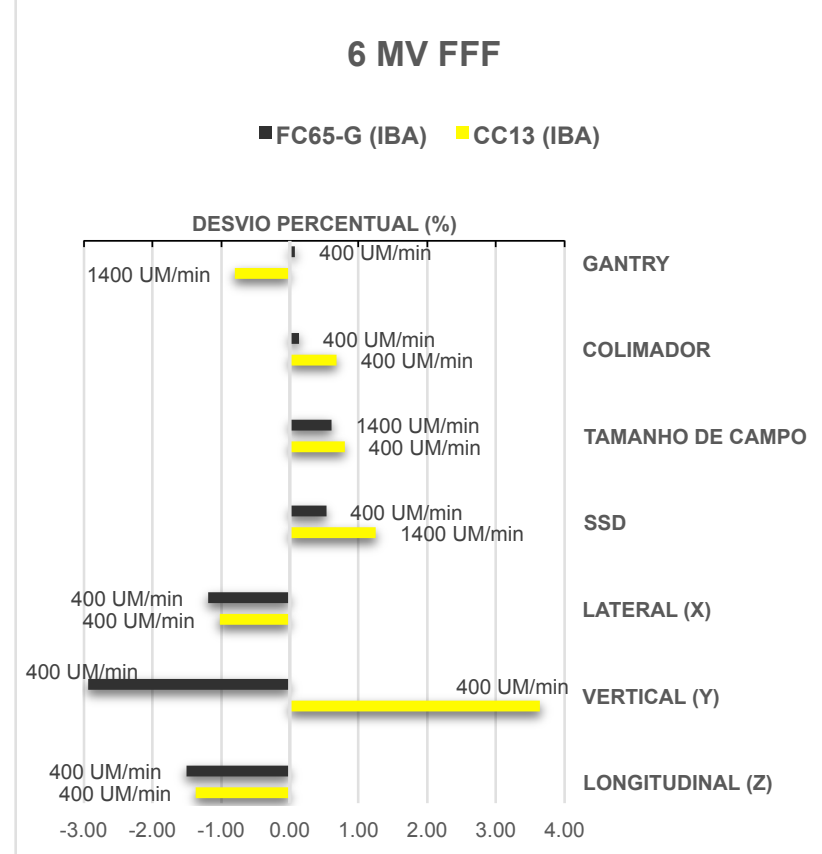

Figura 5. Desvio percentual máximo (\%) encontrado em cada parâmetro, comparando-se dois detectores para o feixe de $6 \mathrm{MV}$ FFF;

\section{FFF}

-FC65-G (IBA) CC13 (IBA)

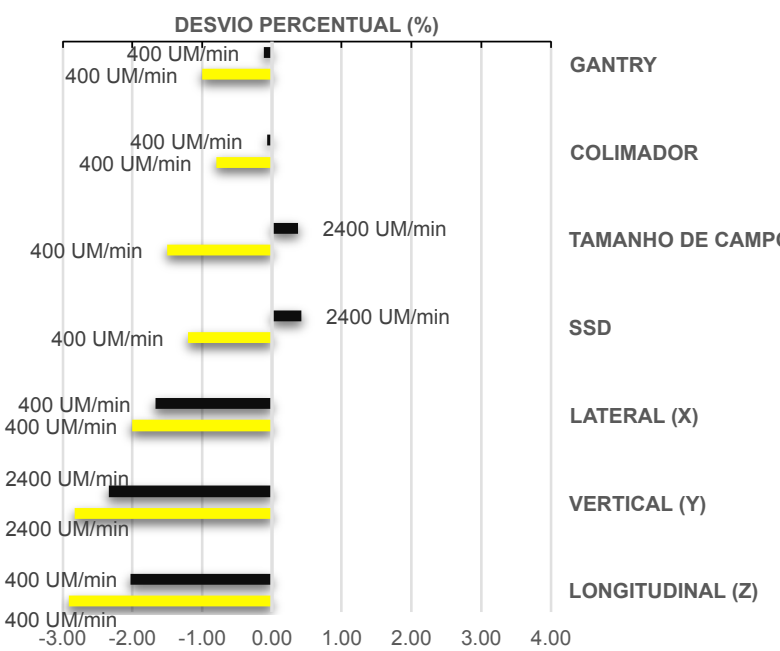

Figura 6. Desvio percentual máximo (\%) encontrado em cada parâmetro, comparando-se dois detectores para o feixe de $10 \mathrm{MV}$ FFF;

Tabela 5. Desvios percentuais máximos, para cada parâmetro avaliado dentro de cada feixe;

\begin{tabular}{ccccc}
\hline Feixe $(\mathrm{MV})$ & \multicolumn{2}{c}{ 6 FFF } & \multicolumn{2}{c}{ 10 FFF } \\
\hline Detector & CC13 & FC65 & CC13 & FC65 \\
\hline Gantry $\left(^{\circ}\right)$ & 0,079 & $-0,797$ & $-0,118$ & $-1,002$ \\
Colimador $\left(^{\circ}\right)$ & 0,132 & 0,683 & $-0,071$ & $-0,802$ \\
Campo $\left(\mathrm{cm}^{2}\right)$ & 0,609 & 0,796 & 0,378 & $-1,503$ \\
SSD $(\mathrm{cm})$ & 0,529 & 1,253 & 0,425 & $-1,202$ \\
Lateral $(\mathrm{X})(\mathrm{cm})$ & $-1,191$ & $-1,024$ & $-1,675$ & $-2,010$ \\
Vertical $(\mathrm{Y})(\mathrm{cm})$ & $-2,939$ & 3,641 & $-2,340$ & $-2,825$ \\
Longitudinal $(\mathrm{Z})(\mathrm{cm})$ & $-1,509$ & $-1,365$ & $-2,029$ & $-2,915$ \\
\hline \hline
\end{tabular}

Para o feixe de 6MV FFF, as posições onde ocorreram os maiores desvios para os detectores FC65-G e CC13 foram, respectivamente: $-1,0^{\circ}$ e $1,0^{\circ}$ para Gantry; $+0,5^{\circ}$ e $-1,0^{\circ}$ para Colimador; 10,4 $\mathrm{cm}^{2}$ e 10,4 $\mathrm{cm}^{2}$ para Tamanho de Campo; $99,8 \mathrm{~cm} \mathrm{e}$ $99,8 \mathrm{~cm}$ para SSD; $-1,0 \mathrm{~cm} \mathrm{e}-1,0 \mathrm{~cm}$ para ambas as posições $X$ e Z; $+0,5 \mathrm{~cm}$ e $-0,5 \mathrm{~cm}$ para posição $Y$;

Para o feixe de $10 \mathrm{MV} F F F$, as posições onde ocorreram os maiores desvios para os detectores FC65-G e CC13 foram, respectivamente: $-0,5^{\circ} \mathrm{e}$ $1,0^{\circ}$ para Gantry; $-1,0^{\circ}$ e $+0,5^{\circ}$ para Colimador; 10,4 $\mathrm{cm}^{2}$ e 9,6 $\mathrm{cm}^{2}$ para Tamanho de Campo; $99,8 \mathrm{~cm}$ e $100,2 \mathrm{~cm}$ para SSD; $-1,0 \mathrm{~cm}$ e $-1,0 \mathrm{~cm}$ para ambas as posições $X$ e $Z ;+0,5 \mathrm{~cm}$ e $+0,5 \mathrm{~cm}$ para posição $\mathrm{Y}$;

A quarta e última análise faz uma avaliação geral de todas as medidas coletadas. Ainda com base no maior desvio percentual (\%) encontrado dentro de cada um dos 7 parâmetros avaliados, dessa vez a análise é feita com referência às leituras coletadas para todas as taxas de dose, feixes e detectores utilizados no experimento. A Figura 7 representa os desvios máximos para cada parâmetro e a Tabela 6 mostra em qual variável (Detector, Taxa de Dose e Feixe) esse desvio ocorreu:

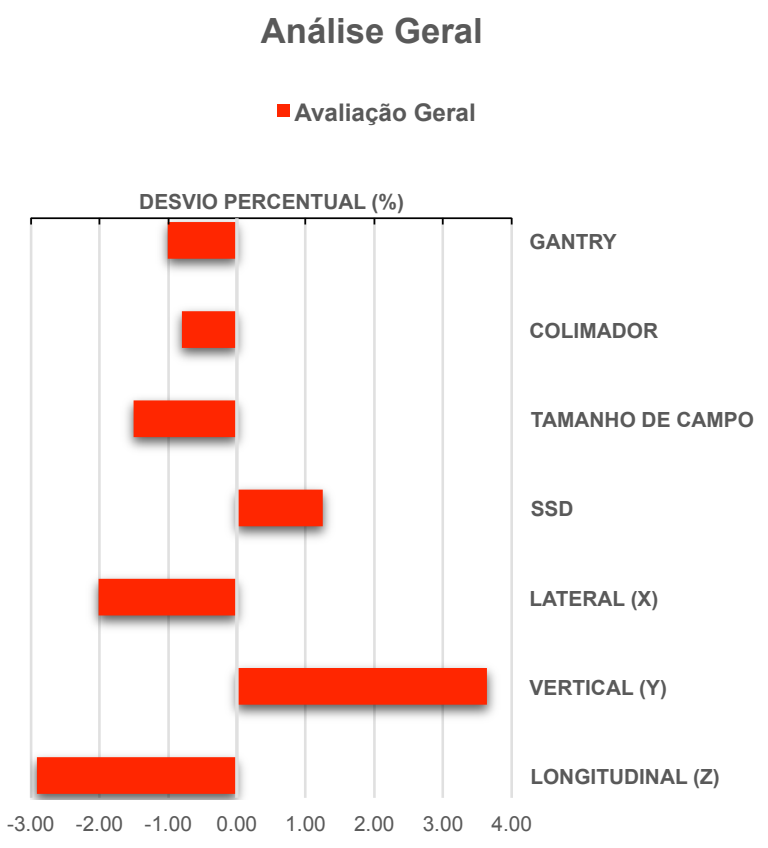

Figura 7. Desvio percentual máximo (\%) encontrado em cada parâmetro, comparando-se todas as variáveis de análise;

Tabela 6. Detector, Taxa de Dose e Feixe onde ocorrem os maiores desvios percentuais, para cada parâmetro avaliado;

\begin{tabular}{clcc}
\hline Parâmetros & Detector & $\begin{array}{c}\text { Taxa de Dose } \\
(\text { UM/min })\end{array}$ & $\begin{array}{c}\text { Feixe } \\
(\text { MV-FFF })\end{array}$ \\
\hline Gantry $\left(^{\circ}\right)$ & CC13 & 400 & 10 \\
Colimador $\left(^{\circ}\right)$ & CC13 & 400 & 10 \\
Campo $\left(\mathrm{cm}^{2}\right)$ & CC13 & 400 & 10 \\
SSD $(\mathrm{cm})$ & CC13 & 1400 & 6 \\
Lateral $(\mathrm{X})(\mathrm{cm})$ & CC13 & 400 & 10 \\
Vertical $(\mathrm{Y})(\mathrm{cm})$ & CC13 & 400 & 6 \\
Longitudinal $(\mathrm{Z})(\mathrm{cm})$ & $\mathrm{CC13}$ & 400 & 10 \\
\hline
\end{tabular}


$\mathrm{Na}$ análise geral, as posições onde ocorreram os maiores desvios foram: $-1,0^{\circ}$ para Gantry; $+0,5^{\circ}$ para Colimador; 9,6 $\mathrm{cm}^{2}$ para Tamanho de Campo; 99,8 $\mathrm{cm}$ para SSD; $-1,0 \mathrm{~cm}$ para ambos desvios em $X$ e Z; -0,5 cm para desvios em Y;

\section{Discussão}

\subsection{Análise por detectores}

$\mathrm{Na}$ primeira análise realizada, nota-se que para erros em parâmetros mecânicos (Gantry, Colimador, Tamanho de Campo e SSD) os desvios ficaram relativamente baixos. Para a FC65-G foi atingido um valor máximo de $0,6 \%$, e para a câmara de ionização CC13, os resultados ficaram abaixo de $1,5 \%$. Nota-se que de forma geral os desvios foram maiores para a CC13, mas ainda assim para ambas as câmaras as incertezas estão dentro dos limites toleráveis especificados pelo TRS-398 e pelo TG142, e individualmente, não iriam comprometer os resultados. Em se tratando de erros de posicionamento do detector, os maiores desvios da série de medidas foram encontrados, sempre com valores acima de $1,5 \%$. Ainda assim a CC13 obteve os maiores desvios em cada posição. Para desvios laterais e longitudinais, todas as situações representaram uma subestimação da dose, que pode ser explicado pelo formato do perfil do feixe, que possui um elevado fall-off de dose fora do eixo central. Para erros na vertical, os desvios ficaram em torno de $3,0 \%$, chegando a atingir $3,6 \%$ para a CC13. De forma geral, o maior impacto dos desvios pode ser atribuído à $\mathrm{CC} 13$, que devido a seu baixo volume acaba sendo mais sensível a qualquer tipo de variação fora das condições ideais. Notou-se ainda uma certa dependência da CC13 com as taxas de dose utilizadas. Para esse detector, os desvios foram maiores na situação onde se utilizou a taxa de dose padrão (400 UM/min).

\subsection{Análise por diferentes taxas de dose}

$\mathrm{Na}$ segunda análise, notou-se mais uma vez um desvio dentro dos limites toleráveis já explanados para os parâmetros mecânicos. Entretanto, notou-se uma forte dependência do feixe utilizado com a taxa de dose: em $400 \mathrm{UM} / \mathrm{min}$ os desvios foram mais evidentes para o feixe de $10 \mathrm{MV} F F F$, e nas maiores taxas de dose ele foi mais evidente para o feixe de 6 MV FFF. Nota-se mais uma vez maiores desvios ocorrendo no posicionamento do detector. Erros laterais e longitudinais mais evidentes quando se usou taxa de dose de $400 \mathrm{UM} / \mathrm{min}$, chegando a quase $3 \%$ para um erro longitudinal utilizando taxa de dose padrão. Desvios verticais ficaram acima de $2 \%$ para ambas taxas de dose, já representando uma situação fora da tolerância mensal padrão, porém notou-se uma evidência de desvio maior para o feixe de 6 MV FFF. Numa avaliação geral, observou-se uma grande tendência de maiores desvios quando se utiliza menores taxas de dose no momento da realização da dosimetria.

\subsection{Análise por diferentes feixes energéticos}

Para a terceira análise, os desvios ficaram dentro dos limites toleráveis em se tratando de parâmetros mecânicos, e os erros de posicionamento do detector foram, mais uma vez, os maiores encontrados dentro dos sete parâmetros. Avaliando dentro da câmara de ionização CC13, os desvios encontrados foram sempre maiores especificamente para o feixe de 10 MV FFF. O mesmo ocorre quando se compara desvios na posição longitudinal e lateral do detector. Isso ocorre devido às características do perfil de dose da curva para o feixe de $10 \mathrm{MV} F F F$, cuja intensidade, em comparação com o feixe de $6 \mathrm{MV}$ FFF é maior no eixo central e tal qual o gradiente de dose é mais acentuado fora desse eixo. O mesmo não se pode dizer dos erros simulados na vertical, tanto representando uma situação de mudança de SSD ou representando uma mudança na profundidade do detector: para esses casos o feixe de 6MV FFF foi o que apresentou os maiores desvios da série de medidas. Isso se deve a dois fatores de extrema importância: o primeiro diz respeito a mudanças em características ligadas à curva em profundidade (PDP), cujo efeito é mais pronunciado a baixas energias. $E$ o segundo já diz respeito a mudanças no fator de recombinação iônica $\left(P_{\text {ion }}\right)$, que mesmo não mensurado está intrínseco nas medidas e é dependente da dose por pulso, sensível a deslocamentos verticais.

\subsection{Análise geral}

A última análise realizada sobre os dados permitiu um olhar mais abrangente do que realmente influencia a dosimetria de referência de feixes FFF. Uma câmara de menor volume (CC13) interfere fortemente nos resultados, pois é mais sensível a erros de setup que estejam fora das condições de referência. Uma taxa de dose baixa também mostrou uma tendência a influenciar a coleta de carga pelo detector, e com isso provocar maiores desvios nas medidas. Altas energias também são um grave problema quando se introduz erros mecânicos e de posicionamento da câmara, em geral pelo perfil mais intenso e de elevado gradiente fora do eixo central, que foi apresentado pelo feixe de 10 MV FFF. A exceção ocorre quando as mudanças dos parâmetros ocorrem na profundidade de medida, como foi o caso das mudanças na SSD e na profundidade da câmara em y, que alteram fortemente características intrínsecas à curva de PDP, cuja sensibilidade a mudanças é maior para baixas energias. Os desvios máximos nas posições lateral e longitudinal do detector sugeriram dados expressivos: diminuição de 2,0\%, chegando a 2,9\% em z, sempre na variação máxima do parâmetro $(1,0$ $\mathrm{cm}$ ). A mesma notoriedade ocorreu para um erro de posicionamento vertical: chegou a um aumento de $3,6 \%$, maior do que valores já encontrados em outros estudos ${ }^{8}$ que trabalharam com feixes convencionais (FF) e encontraram o maior desvio também no posicionamento vertical do detector, porém com valores máximos de 3,0\%. 


\section{Conclusões}

De forma geral, concluiu-se desse trabalho que a montagem do setup experimental para a dosimetria de referência para feixes em modo FFF deve ser feita de forma cautelosa e com muita atenção, a fim de se evitar problemas na calibração da máquina e, com isso, gerar problemas na entrega da dose ao paciente. Deve-se atentar que quando a dosimetria for executada para maiores energias (10 MV FFF), câmara de ionização de baixo volume (CC13) e utilizando baixa taxa de dose (400 UM/Min), uma maior atenção deve ser dada à montagem do setup. Apenas para o feixe de 6MV FFF, um cuidado maior deve ser tomado para possíveis erros verticais. Com isso, um rígido programa de controle de qualidade deve garantir os parâmetros mecânicos do aparelho, cujos desvios mesmo estando dentro do tolerável ainda podem ser somados entre si ou entre outros fatores e gerar maiores impactos.

\section{Agradecimentos}

Agradecimentos especiais à toda equipe do setor de Radioterapia do Hospital de Amor de Barretos, incluindo físicos titulares, médicos, dosimetristas e residentes, pelo apoio dedicado a esse projeto.

\section{Referências}

1. David Thwaites. Accuracy required and achievable in radiotherapy dosimetry: have modern technology changed our views? Journal of Physics: Conference Series 444, 2013.

2. Klein EE, Hanley J, Bayouth J, Yin FF, Simon W, Dresser S. Task Group 142 Report: Quality Assurance of Medical Accelerators; American Association of Physicists in Medicine, 2009.

3. Andreo P, Burns DT, Hohlfeld K, Huq MS, Kanai T, Laitano F. International Atomic Energy Agency (IAEA). Absorbed Dose Determination in External Beam Radiotherapy. Technical Report Series n. 398. Vienna; IAEA; 2000.

4. Kry SF, Popple R, Molineu A, Followill DS. Ion recombination correction factors $\left(P_{i o n}\right)$ for Varian TrueBeam high-dose-rate therapy beams. Journal of Applied Clinical Medical Physics, Vol 13, N. 6, 2012.

5. McEwen M, DeWerd L, Ibbott G, Followill D, Rogers DWO, Seltzer $S$ et al. Addendum to the AAPM's TG-51 protocol for clinical reference dosimetry of high energy photon beams. American Association of Physicists in Medicine, 2014.

6. Ruggieri R, Naccarato S, Stavrev P, Syavreva N, Pasetto S, Salamone I et al. Correction for intra-chamber dose gradients in reference dosimetry of flattening-filter-free MV photon beams. American Association of Physicists in Medicine, 2016.

7. Xiao Y, Kry SF, Popple R, Yorke E, Papanikolaou N, Stathakis $S$ et tal. Flattening Filter-Free Accelerators: a report from the AAPM Therapy Emerging Technology Assessment Work Group. Journal of Applied Clinical Medical Physics, Vol 16, n. 3, 2015.

8. Radicchi LA, Baptista LS, Silva PPCE. Influence analyzes of the variations on quality control parameters in determination of absorbed dose in water. Revista Brasileira de Física Médica, 4(1); 27-30; 2010.

\section{Contato:}

Gustavo Costa Panissi

Rua Antenor Duarte Villela, 1331 - Dr. Paulo Prata, Barretos -SP, 14784-400.

E-mail: panissi.gustavo@gmail.com 